\title{
Visual Assessment of Coronary Artery Calcium in Non-ECG Gated Chest CT: A Multicenter Study
}

Hideya Yamamoto ( $\nabla$ hideyayamamoto-circ@umin.ac.jp )

Yasuda Women's University https://orcid.org/0000-0001-6357-1896

\section{Shinichiro Fujimoto}

Juntendo University: Juntendo Daigaku

\section{Chihiro Aoshima}

Juntendo University: Juntendo Daigaku

\section{Tohru Minamino}

Juntendo University: Juntendo Daigaku

\section{Takashi Fujii}

JA Hiroshima General hospital

\section{Shinichi Wakabaayshi}

Suideikai Kajikawa Hospital

\section{Yoji Urabe}

Hiroshima Prefectural Hospital: Kenritsu Hiroshima Byoin

\section{Hironori Ueda}

Hiroshima Prefectural Hospital: Kenritsu Hiroshima Byoin

\section{Eiji Kunita}

Hiroshima city asa Hospital

\section{Mitsunori Abe}

Matsuyama Heart Center Yotsuba Clinic

\section{Hiroshi Higashino}

Matsuyama Heart Center Yotsuba Clinic

\section{Research article}

Keywords: Chest CT, Coronary artery calcium, Weston score, Slice number, Agreement, Diagnostic power.

Posted Date: July 19th, 2021

DOI: https://doi.org/10.21203/rs.3.rs-439043/v2

License: (c) (1) This work is licensed under a Creative Commons Attribution 4.0 International License. Read Full License 


\section{Abstract}

Purpose: We sought to evaluate the visual measurements of coronary artery calcium (CAC) in chest CT without ECG gating, and to compare their predictive abilities for obstructive coronary artery disease (CAD).

Methods: We analyzed 163 subjects who underwent both coronary and chest CT examinations at 6 centers within 3 months. Agatston scores were calculated on standard ECG-gated scans and then classified into none (0), mild (1-99), moderate (100-400), and severe (>400) grades. Chest CT images were reconstructed with standard $5.0 \mathrm{~mm}$ axial slices. CAC in chest CT scans was measured using two methods: Weston score (sum of the assigned score of each vessel, range: 0-12) and number of slices showing CAC (Ca-slice\#).

Results: We found excellent inter-observer agreement in the estimates of the Weston score and Ca-slice\# $\left(R^{2}=0.913\right.$ and 0.955 , respectively). When each of the two scores was divided into four levels, good agreement with the 4-grade Agatston score was observed (kappa value $=0.604$ and 0.794 , respectively). Of the 155 patients remaining after excluding non-diagnostic studies, obstructed CAD, defined as the presence of $\geq 70 \%$ stenosis on CT angiography, was found in 43 (27\%). Receiver-operator characteristic curves of 4-grade hierarchies of Agatston score, Weston score, and Ca-slice\# provided similar diagnostic powers to detect obstructed $\operatorname{CAD}(0.722,0.706$, and 0.718 , respectively).

Conclusion: The visual assessments of CAC detected by ECG non-gated chest CT scans were in good agreement with the ECG-gated Agatston score and also provided equivalent power to detect obstructive CAD.

This study was registered at UMIN-CTR (identifier: UMIN000039178; registered date; Jan 16, 2020)

\section{Introduction}

Coronary artery calcium score is associated with the prevalence of coronary artery disease (CAD) [1, 2], mortality, and morbidity $[3,4]$. Coronary artery calcification (CAC) is screened by coronary computed tomography (CT) using the ECG-gated method, but is also often found incidentally on chest CT examinations. CAD is often complicated in patients with respiratory diseases such as lung cancer [5] or chronic obstructive pulmonary disease [6] because of the existing coronary risk factors of old age and cigarette smoking.

Several reports show that CAC on chest CT without ECG gate is in good agreement with the CAC score detected by the ECG-gated method [7-9]. In addition, visual estimation of CAC is expected to be an easy and feasible method for detecting CAD in subjects who undergo chest CT examinations. Several evaluation methods have been reported for the visual measurement of CAC in chest CT [10-13]; however, no established methods have yet been recommended. 
In this multicenter study, we sought to validate the usefulness of visual estimation of CAC score in chest CT examinations without an ECG gated method, and to compare the predictive abilities of obstructive CAD using these visual CAC scoring systems in suspected CAD patients.

\section{Methods}

\section{Study design}

Between April and December 2020, patients who had an indication for coronary CT angiography were prospectively eligible for participation in this study to undergo simultaneous chest CT examination, but if patients had undergone chest CT examination to examine chest diseases performed within $<3$ months before, these images were used for analysis. Patients were excluded from participation in the trial for the following reasons: body mass index $(\mathrm{BMI}) \geq 35 \mathrm{~kg} / \mathrm{m}^{2}$ (because image quality was well guaranteed even in patients with a BMl of $30-35 \mathrm{~kg} / \mathrm{m}^{2}$, the criterion was amended from $30 \mathrm{~kg} / \mathrm{m}^{2}$ ), $\mathrm{known} C A D$, prior treated structure heart disease, status acute coronary syndrome or other urgent status, severe heart failure status, prior device implantation, and inadequate image quality. The study was performed at six centers in Japan (Supplemental Table 1). The ethical review board of Yasuda women's university and each participating institution approved the study protocol. Written informed consent was obtained from all patients. This study was registered at UMIN-CTR (identifier: UMIN000039178).

\section{Coronary CT scan protocol and Agatston score}

Coronary CT examinations were performed using new generation-type multidetector CT scanners according to each hospital's scan protocol (Supplemental Table 1). Prior to a contrast-enhanced scan, a non-contrast coronary CT scan was performed to detect the CAC. The following parameters were unified among hospitals: tube voltage, $120 \mathrm{kV}$; FOV, $25 \mathrm{~cm}$. Thirty-five to 40 contiguous non-contrast coronary images of 2.5- (GE scanner) or 3.0- (Cannon scanner) mm thickness were obtained at 75\% RR intervals. CAC scores on ECG-gated coronary CT were calculated using the conventional Agatston method in each hospital [1]. Agatston CAC was categorized into the following four classes: none $=0$, mild $=1-99$, moderate $=100-400$, and severe $>400[14]$.

A contrast-enhanced coronary CT scan was performed according to the SCCT guideline [15]. If the patient's resting heart rate was $>65$ beats per minute, a $\beta$-blocker (metoprolol $20-40 \mathrm{mg}$ ) was administered orally 60 min before the CT scan to avoid motion artifacts. The results of coronary CT were assessed in each hospital according to the criteria of the CAD-RADS reporting system [16]. Obstructive CAD was assessed by the presence of $\geq 70 \%$ diameter stenosis in any coronary artery or $\geq 50 \%$ diameter stenosis in the left main coronary artery [16].

\section{Chest CT scan and evaluation of non-ECG gated CAC}


Chest CT examinations without ECG gating were performed at each center using the same model as coronary CT examinations, including imaging of all lung fields from the apex to the base. The tube voltage of the chest CT scan was uniformly set at $120 \mathrm{kV}$. Chest CT images were reconstructed with standard $5.0 \mathrm{~mm}$ axial slices.

\section{Data analysis of CAC on chest CT}

Chest CT images performed at each center were uploaded and saved as an anonymized DICOM format file in a specific "image storage system", installed on the cloud. Visual assessment of CAC on chest CT images of enrolled patients was performed by two skilled interpreters who were blinded to the patient information (SF and $\mathrm{HH}$ ). Images were interpreted using free software (Sycorax DICOM viewer, CodeDynamix Co., Ltd), setting window levels of 35 to 50 Hounsfield units, and a window width of 350 to 500 Hounsfield units. Visual assessment was performed using two different methods. First, the Weston score was calculated according to a previous report [8-10]. The four coronary arteries (left main, left anterior descending, left circumflex, and right coronary) were scored for the extent of calcification using a 4-point scale: 0 = no calcium, 1 = one pixel of calcium, 2 = more than one pixel, but insufficient to classify as 3; and 3 = hard calcium, as evidenced by blooming artifact. We also used a unique evaluation method to count the number of slices containing CAC (Ca-slice\#).

Interobserver agreement of each visual CAC measurement between the two readers was assessed in 40 randomly selected patients. Figure 1 shows a representative case of coronary calcium images on chest CT examination.

\section{Statistical analysis}

The prevalence of obstructive CAD in the registered samples was estimated to be $25 \%$. The required number of cases was calculated to be 95 when the area under the receiver-operator characteristic curves was estimated to be 0.71 , the detection rate was 0.9 , and the significance level was 0.05 , which indicates the good predictive ability of obstructive CAD by the qualitative calcium evaluation method. Furthermore, we estimated the exclusion of test cases due to clinical reasons and imaging problems to be $10 \%$, and set the target minimum number of cases to 106.

Categorical variables are reported as numbers (\%). Continuous variables with a normal distribution are presented as mean \pm SD, and Agatston scores with a skewed distribution are presented as median and range. Agreements between two visually estimated measurements (Weston score and Ca-slice\#) were tested using Pearson's correlation and Bland-Altman plots. Visual measurements were categorized into four classes (no, mild, moderate, and severe) depending on the Weston score. Then Agatston classes with the greatest frequency of scores were grouped in Weston classes. Agreements of score classes were expressed as Cohen's kappa statistics (95\% confidence interval [Cl]). A kappa value of $>0.8$ indicated excellent agreement, and a kappa value of 0.60 to 0.80 indicated good agreement. 
Using receiver-operating characteristic (ROC) curves, c-statistics were determined for each calcium measurement to diagnose obstructive CAD. The optimal cut-off point was obtained from the Youden index [maximum (sensitivity+ specificity -1)]. Diagnostic measures were calculated, including sensitivity, specificity, positive predictive value, negative predictive value, and accuracy. All statistical analyses were performed using JMP Pro Version 15 and SAS version 9.4.2 (SAS Institute, Cary, NC). Statistical significance was set at $P<0.05$.

\section{Results}

\section{Eligible patients}

In this prospective multicenter study, 165 patients were initially eligible, and 2 were excluded because of known CAD or poor image quality. A total of 163 patients from six institutions were analyzed, including: Kajikawa Hospital ( $n=29)$, Juntendo University $(n=40)$, JA Hiroshima Hospital $(n=30)$, Hiroshima Prefectural Hospital $(n=21)$, Hiroshima City Asa Hospital $(n=12)$, and Matsuyama Heart Center $(n=30)$. The average age was $68.4 \pm 12.9$ years, and 99 (61\%) were men. Seventy-four (46\%) patients complained of atypical chest pain and $28(17 \%)$ complained of typical chest pain, whereas the remaining $61(37 \%)$ patients had no chest symptoms. The study sample included $28(17 \%)$ patients with cerebral infarction and eight (5\%) patients with peripheral artery disease.

Chest CT examinations were prospectively performed in 105 patients $(65 \%)$, and coronary CT scans were performed simultaneously. In the remaining 58 patients, chest CT examinations performed within 79 days before coronary CT [43 (27\%) cases were performed within 1 to 7 days] were used for analysis. The Agaston score ranged from 0 to 3166 (median, 90). The prevalence of obstructive CAD was 43 (27\% patients) (Table 1).

\section{Interobserver agreements of each visual CAC measurement}

There was an excellent interobserver agreement of each CAC measurement between the two observers $\left(R^{2}=0.913\right.$ and 0.955 , Weston score, and Ca-slice\#, respectively) (Supplemental Figure 1).

\section{Agreements between visual CAC measurement and Agatston score}

In the cohort, $20 \%, 32 \%, 22 \%$, and $27 \%$, had none (0), mild (1-99), moderate (100-400), and severe (>400) Agatston classes, respectively.

Figure 2 shows the distribution of Agatson classes by Weston score (0-12) (Panel A) and by Ca-slice\# (0-17) (Panel B). Weston score was classified according to the hierarchy of the Agatston score, and graded as none, 0 ; mild, 1-4; moderate, 5-8; and severe, 9-12. The Weston score class was in good 
agreement with the Agatston score class [kappa value $=0.604$ (95\% Cl 0.511-0.696)] (Panel C). Similarly, Ca-slice\# was graded as none $=0$, mild $=1-4$, moderate $=5-8$, and severe $=9-17$. Ca-slice\# also showed good agreement with the Agatston score class [kappa value $=0.794(95 \% \mathrm{Cl} 0.720-0.869)$ ] (Panel D).

Weston score classes agreed with Agatston score classes in $115(71 \%)$ patients, but disagreed with the remaining $48(29 \%)$ patients. The Ca-slice\# classes agreed with Agatston score classes in $138(85 \%)$ patients, but disagreed with the remaining $25(15 \%)$ patients. In each visual score, differences in clinical and imaging factors and CT machines were compared between the agreed and disagreed groups, but no significant parameters affecting their agreements were found.

\section{Diagnostic performance for obstructive CAD using non-ECG gated calculated and visual estimated CAC scores}

After excluding 8 patients with non-diagnostic studies, the diagnostic power was evaluated in 155 patients. Obstructed CAD was found in 43 patients (27\%).

ROC analyses (Figure 3) showed comparisons of diagnostic performance in detecting obstructive CAD using three scores. The C-statistics of Agatston score, Weston score, and Ca-slice\# were 0.750 (95\% Cl 0.646-0.831), 0.721 (95\% Cl 0.618-0.805), and 0.706 (95\% Cl 0.603-0.792), respectively ( $p=$ 0.046 ). Ca-slice\# had a significantly lower c-statistic than the Agatston score (difference 0.044 [95\% $\mathrm{Cl} 0.008-0.080$ ], $P=0.016)$.

In comparison, when divided into four classes, the c-statistics of 4 the 4-grade hierarchies of Agatston score, Weston score, and Ca-slice\# were 0.722 ( $95 \% \mathrm{Cl} 0.587-0.826), 0.706$ (95\% Cl, 0.609-0.781), and $0.718(95 \% \mathrm{Cl} 0.618-0.801)$, respectively, with no statistically significant differences $(P=0.798)$.

When each cutoff point was set at an Agatston score of 179, Weston score of 6 , and Ca-slice\# of 4, the sensitivity and specificity, respectively, for identifying obstructive CAD were $70 \%$ and $79 \%$ for Agatston score, $65 \%$ and $74 \%$ for Weston score, and $81 \%$ and $61 \%$ for Ca-slice\#. When the cutoff point was set at a moderate class of each score, corresponding to an Agatston score of $\geq 100$, Weston score of $\geq 6$, and Caslice\# of $\geq 4$, the sensitivity and specificity, respectively, for identifying obstructive CAD were $79 \%$ and $63 \%$ for Agatston score, $84 \%$ and $51 \%$ for Weston score, and $81 \%$ and $61 \%$ for Ca-slice\# (Table 3).

\section{Discussion}

This study was conducted in collaboration at six centers in Japan to evaluate the usefulness of visual measurements of CAC detected by ECG non-gated chest CT scans. Our results showed that two visual measurements of CAC detected by chest CT scans were in good agreement with the ECG-gated Agatston score. The first method studied was the Weston score, which has already been reported, and the other is a simple method to count the number of slices in which calcium is reflected (Ca-slice\#). These scores were 
in good agreement with the Agatston score obtained by the ECG-gate, using grading into four classes. In addition, the prediction rates of obstructive CAD detected on coronary CT angiography were equivalent to the Agatston score obtained by ECG-gate coronary CT.

\section{Predictive ability of Weston score}

ECG non-gated CAC scoring has become widespread as an easier screening method for CAD. The Weston score is a visual scoring method that includes segmented vessel-specific scoring using an ordinal scale of $0-3$ [12]. Based on previous reports [8-10], Weston scores of $\geq 7$, which correspond to an Agatson score of $\geq 400$, were defined as clinically significant. In a large multicenter cohort study, Weston scores of $\geq 7$ indicated an elevation in predicting incident CAD in smokers [10]. Chandra et al. reported that the Weston score was categorized into four groups as follows: $0,1-2,3-7$, and $>8$ [9]. Using this category criterion for our data, both classes showed moderate agreement [kappa value of 0.574 ( $95 \% \mathrm{Cl} 0.480-$ 0.668)] (Supplemental Figure 2). However, the divisional levels of the moderate and severe groups were defined as 4 and 9, respectively, which were higher than those reported previously; this change also improved the kappa value from 0.574 to 0.706 .

It has also been reported that the Weston score has a high diagnostic ability to detect obstructive CAD. For example, Bhatt et al reported that, for predicting prevalent CAD, c-statistics for the Weston and Agatston scores were 0.78 and 0.74 , respectively [11], and West et al reported that a Weston score of 6 maximized the sensitivity and specificity at $89 \%$ and $76 \%$, respectively [12]. Our data showed that the cstatistic for the Weston score was 0.750 , and when the optimal cutoff point was set at 6 , the sensitivity and specificity were $65 \%$ and $74 \%$, respectively.

\section{Predictive ability of Ca-slice\#}

In the present study, we proposed that Ca-slice\#, counts of slices showing CAC as a new visual CAC measurement. The number of calcium slices is a highly reproducible and easily measurable index in chest CT taken with the same $5 \mathrm{~mm}$ slice, which is frequently used in clinical diagnosis. When Ca-slice\# was graded into four classes, it also showed good agreement with the Agatston score class (kappa value $=0.794)$. In addition, although the predictive ability of Ca-slice\# was inferior to the Agatson score, when both scores were graded into four classes, the predictive ability of Ca-slice\# improved to be equivalent to the Agatson score.

Ca-slice\#, as an index, may include several problems, such as the size of the heart according to the physique, concentration of calcium deposit, or anatomical features of the coronary artery. For example, the proximal part of the left anterior descending artery, which is a frequent site of calcification, runs in the horizontal direction, while the proximal part of the middle part of the right coronary artery runs in the axial direction. Therefore, calcification in the former is underestimated, while in the latter, it is overestimated. The diagnostic value is expected to increase further if these problems can be corrected. 


\section{Study limitations}

First, this study was conducted in a cross-sectional manner to evaluate whether visual CAC scoring had predictive abilities to determine obstructive CAD on coronary CT angiography. The predictive power of hard outcomes was not assessed. Therefore, in the future, it will be necessary to verify the usefulness of visual evaluation for predicting long-term prognosis. Second, the diagnostic power of Ca-slice\# was significantly lower than that of the Agatston score (c-statistics: 0.750 vs. 0.706 , difference: $0.044, P=$ 0.016). However, when Ca-slice\# was graded into four classes, it provided a good agreement with the Agatston score class (kappa value $=0.794$ ). However, we could not find any significant parameters affecting the disagreements of score classes. To improve the diagnostic power, we plan to develop a new scoring system using machine learning adding methods [17]. Finally, in this study, chest CT images were reconstructed with $5.0 \mathrm{~mm}$ axial slices. It is also necessary to measure the scores with different scan widths [18].

\section{Conclusion}

From our results in six centers, the visual assessments of CAC detected by ECG non-gated chest CT scan, Weston score, and Ca-slice\# were in good agreement with the ECG-gated Agatston score. In addition, they also have a similar predictive power of obstructive CAD to the Agatston score.

\section{Declarations}

\section{Financial support}

This study was funded by a Grant-in-Aid for Scientific Research from Yasuda Women's University (No.102549).

\section{Conflict of interest:}

Shinichiro Fujimoto received grants from HeartFlow Japan G.K, Canon Medical Systems Corporation, and FUJIFILM Corporation, Edwards LifeSciences Ltd. All other authors declare no competing interests.

\section{Ethics approval}

The ethical review board of Yasuda women's university (No. 190011) and each participating institution approved the study protocol.

\section{Consent to participate}

Written informed consent was obtained from all patients.

\section{Acknowledgement}


We would like to thank Katsuyuki Haino (Biomet Corporation, Tokyo) for statistical analysis, and Editage (www.editage.com) for English language editing.

\section{Participating Institutions and Investigators}

JA Hiroshima University; Shuji Tsujiyama, Hajime Shimodoi; Kajikawa Hospital; Eiji Imamura, Hiroki Sasaki; Matsuyama Heart Center Yotsuba Circulation Clinic, Fumitaka Kawajo, Aya Kashima; Hiroshima Prefectural Hospital; Naoya Mitsuba, Shogo Matsui, Masashi Takahashi, Satoshi Inada.

\section{References}

1. Agatston AS, Janowitz WR, Hildner FJ, Zusmer NR, Viamonte M Jr, Detrano R (1990) Quantification of coronary artery calcium using ultrafast computed tomography. J Am Coll Cardiol 15:827-832. https://doi.org/10.1016/0735-1097(90)90282-t.

2. Budoff MJ, Georgiou D, Brody A, Agatston AS, Kennedy J, Wolfkiel C, Stanford W, Shields P, Lewis RJ, Janowitz WR, Rich S, Brundage BH (1996) Ultrafast computed tomography as a diagnostic modality in the detection of coronary artery disease: a multicenter study. Circulation 93:898-904. https://doi.org/10.1161/01.cir.93.5.898.

3. Detrano R, Guerci AD, Carr JJ, Bild DE, Burke G, Folsom AR, Liu K, Shea S, Szklo M, Bluemke DA, O'Leary DH, Tracy R, Watson K, Wong ND, Kronmal RA (2008) Coronary calcium as a predictor of coronary events in four racial or ethnic groups. N Engl J Med 358:1336-1345. https://doi.org/10.1056/NEJMoa072100.

4. Yeboah J, McClelland RL, Polonsky TS, Burke GL, Sibley CT, O'Leary D, Carr JJ, Goff DC, Greenland P, Herrington DM (2012) Comparison of novel risk markers for improvement in cardiovascular risk assessment in intermediate-risk individuals. JAMA 308:788-795. https://doi.org/ 10.1001/jama.2012.9624.

5. Janssen-Heijnen ML, Schipper RM, Razenberg PP, Crommelin MA, Coebergh JW (1998) Prevalence of co-morbidity in lung cancer patients and its relationship with treatment: a population-based study. Lung Cancer 21:105-113. https://doi.org/10.1016/s0169-5002(98)00039-7.

6. Chen W, Thomas J, Sadatsafavi M, FitzGerald JM (2015) Risk of cardiovascular comorbidity in patients with chronic obstructive pulmonary disease: a systematic review and meta-analysis. Lancet Respir Med 3:631-639. https://doi.org/10.1016/S2213-2600(15)00241-6.

7. Budoff MJ, Nasir K, Kinney GL, Hokanson JE, Barr RG, Steiner R, Nath H, Lopez-Garcia C, Black-Shinn J, Casaburi R (2011) Coronary artery and thoracic calcium on noncontrast thoracic CT scans: comparison of ungated and gated examinations in patients from the COPD gene cohort. $J$ Cardiovasc Comput Tomogr 5:113-118. https://doi.org/10.1016/j.jcct.2010.11.002.

8. Kirsch J, Buitrago I, Mohammed TL, Gao T, Asher CR, Novaro GM (2012) Detection of coronary calcium during standard chest computed tomography correlates with multi-detector computed tomography coronary artery calcium score. Int J Cardiovasc Imaging 28:1249-1256. https://doi.org/10.1007/s10554-011-9928-9. 
9. Chandra D, Gupta A, Leader JK, Fitzpatrick M, Kingsley LA, Kleerup E, Haberlen SA, Budoff MJ, Witt M, Post WS, Sciurba FC, Morris A (2017) Assessment of coronary artery calcium by chest CT compared with EKG-gated cardiac CT in the multicenter AIDS cohort study. PLOS ONE 12:e0176557. https://doi.org/10.1371/journal.pone.0176557.

10. Chiles C, Duan F, Gladish GW, Ravenel JG, Baginski SG, Snyder BS, DeMello S, Desjardins SS, Munden RF, NLST Study Team (2015) Association of coronary artery calcification and mortality in the National Lung Screening Trial: A comparison of three scoring methods. Radiology 276:82-90. https://doi.org/10.1148/radiol.15142062.

11. Bhatt SP, Kazerooni EA, Newell JD Jr, Hokanson JE, Budoff MJ, Dass CA, Martinez CH, Bodduluri S, Jacobson FL, Yen A, Dransfield MT, Fuhrman C, Nath H, COPD Gene Investigators (2018) Visual estimate of coronary artery calcium predicts cardiovascular disease in COPD. Chest 154:579-587. https://doi.org/10.1016/j.chest.2018.05.037.

12. West BH, Low CG, Bista BB, Yang EH, Vorobiof G, Busuttil RW, Budoff MJ, Elashoff D, Tobis JM, Honda HM (2019) Significance of coronary artery calcium found on non-electrocardiogram-gated computed tomography during preoperative evaluation for liver transplant. Am J Cardiol 124:278284. https://doi.org/10.1016/j.amjcard.2019.04.025.

13. Xie X, Zhao Y, Geertruida H, de Bock GH, de Jong PA, Mali WP, Oudkerk M, Vliegenthart R (2013) Validation and prognosis of coronary artery calcium scoring in nontriggered thoracic computed tomography: systematic review and meta-analysis. Circ Cardiovasc Img 6:514-521. https://doi.org/10.1161/CIRCIMAGING.113.000092.

14. Greenland P, Blaha MJ, Budoff MJ, Erbel R, Watson KE (2018) Coronary calcium score and cardiovascular risk. J Am Coll Cardiol 72:434-447. https://doi.org/10.1016/j.jacc.2018.05.027.

15. Raff GL, Abidov A, Achenbach S, Berman DS, Boxt LM, Budoff MJ, Cheng V, DeFrance T, Hellinger JC, Karlsberg RP (2009) Society of Cardiovascular Computed Tomography. SCCT guidelines for the interpretation and reporting of coronary computed tomographic angiography. J Cardiovasc Comput Tomogr 3:122-136. https://doi.org/10.1016/j.jcct.2009.01.001.

16. Cury RC, Abbara S, Achenbach S, Agatston A, Berman DS, Budoff MJ, Dill KE, Jacobs JE, Maroules CD, Rubin GD, Rybicki FJ, Schoepf UJ, Shaw LJ, Stillman AE, White CS, Woodard PK, Leipsic JA (2016) CAD-RADS(TM) coronary artery disease - Reporting and data system. An expert consensus document of the Society of Cardiovascular Computed Tomography (SCCT), the American College of Radiology (ACR) and the North American Society for Cardiovascular Imaging (NASCl). Endorsed by the American College of Cardiology. J Cardiovasc Comput Tomogr 10:269-281. https://doi.org/10.1016/j.jcct.2016.04.005.

17. van Velzen SGM, Lessmann N, Velthuis BK, Bank IEM, van den Bongard DHJG, Leiner T, de Jong PA, Veldhuis WB, Correa A, Terry JG, Carr JJ, Viergever MA, Verkooijen HM, Išgum I (2020) Deep learning for automatic calcium scoring in CT: validation using multiple cardiac CT and chest CT protocols. Radiology 295:66-79. https://doi.org/10.1148/radiol.2020191621. 
18. Hughes-Austin JM, Dominguez A 3rd, Allison MA, Wassel CL, Rifkin DE, Morgan CG, Daniels MR, Ikram U, Knox JB, Wright CM, Criqui MH, Ix JH (2016) Relationship of coronary calcium on standard chest CT scans with mortality. JACC Cardiovasc Imaging 9:152-159. https://doi.org/10.1016/j.jcmg.2015.06.030.

\section{Tables}

Table 1 Patients' characteristics

\begin{tabular}{ll} 
Variables & $n=163$ \\
\hline Age (yrs) & $68.4 \pm 12.9$ \\
\hline Men & $99(61 \%)$ \\
\hline Body mass index $\left(\mathrm{kg} / \mathrm{m}^{2}\right)$ & $24.3 \pm 4.6$ \\
\hline Hypertension, yes & $108(67 \%)$ \\
\hline Dyslipidemia, yes & $116(59 \%)$ \\
\hline Diabetes mellitus, yes & $46(29 \%)$ \\
\hline Current smoker, yes & $29(18 \%)$ \\
\hline Chest symptom, no/ atypical/ typical & $61(37 \%) / 74(46 \%) / 28(17 \%)$ \\
\hline Cerebral infarction, yes & $28(17 \%)$ \\
\hline Peripheral artery disease, yes & $8(5 \%)$ \\
\hline Heart rate at rest ECG (bpm) & $68.6 \pm 12.0$ \\
\hline Heart rate at coronary CT examination (bpm) & $59.5 \pm 9.2$ \\
\hline Interval between coronary and chest CT scans & \\
\hline 0 days & $105(65 \%)$ \\
\hline -7 days & $43(27 \%)$ \\
\hline 8-79 days & $15(9 \%)$ \\
\hline Reason for chest CT examination & \\
\hline Prospectively eligible & $98(60 \%)$ \\
\hline Previous chest screening & $56(56 \%)$ \\
\hline Evaluation for proven lung disease & $5(3 \%)$ \\
\hline Evaluation for proven thoracic vascular disease & $4(2 \%)$ \\
\hline
\end{tabular}

$\mathrm{CT}$, computed tomography. 
Table 2 Coronary CT examination

\begin{tabular}{|c|c|}
\hline Variable & $n=163$ \\
\hline Agatston score (median, range) & $90(11-426)$ \\
\hline \multicolumn{2}{|l|}{ CAD-RADS grade ,n(\%) } \\
\hline 0 & $23(14 \%)$ \\
\hline 1 & $36 \quad(22 \%)$ \\
\hline 2 & $40 \quad(25 \%)$ \\
\hline 3 & $13 \quad(8 \%)$ \\
\hline 4 & $33(20 \%)$ \\
\hline 5 & $10 \quad(6 \%)$ \\
\hline \multirow[t]{2}{*}{$\mathrm{N}$} & $8 \quad(5 \%)$ \\
\hline & $n=155^{\star}$ \\
\hline Obstructive $C A D^{\dagger}$ & $43(27 \%)$ \\
\hline
\end{tabular}

CAD, coronary artery disease; CAD-RADS, Coronary Artery Disease-Reporting and Data System * 0 f 13 patients in CAD-RADS ' $N$ ' group, 5 patients were included to obstructive CAD because of having one or more significant stenosis. ${ }^{\dagger}$. defined as the presence of $\geq 70 \%$ diameter stenosis in any coronary artery or $\geq 50 \%$ diameter stenosis in the left main coronary artery.

Table 3 Diagnostic performance of each score to detect obstructed coronary artery disease

Variable

Agatston score

Weston score

Ca-slice\#

Agatston score 4 classes

Weston score 4 classes $\geq$ moderate (4)

Ca-slice\# 4 classes
Optimal cutoff point
Sensitivity Specificity PPV NPV Accuracy

$\begin{array}{lllll}70 \% & 79 \% & 57 \% & 87 \% & 77 \%\end{array}$

$\begin{array}{lllll}65 \% & 74 \% & 49 \% & 85 \% & 72 \%\end{array}$

$\begin{array}{llllll}4 & 81 \% & 61 \% & 44 \% & 89 \% & 66 \%\end{array}$


Ca-slice\#, number of slices showing coronary artery calcium; NPV, negative predictive value; PPV, positive predictive value

\section{Figures}

Fig.1
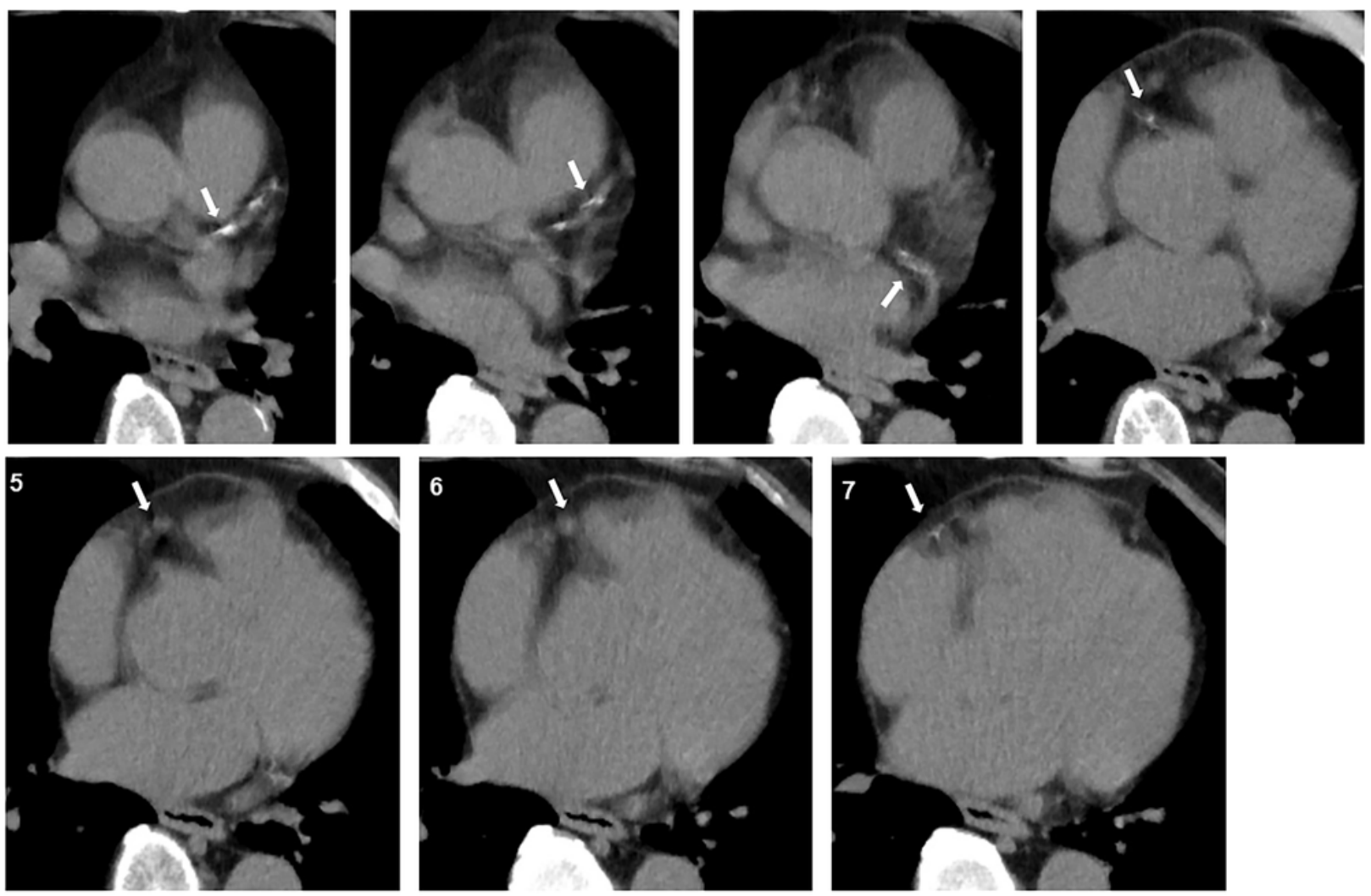

\section{Figure 1}

A representative case A 69-year-old asymptomatic man had hypertension and a history of cerebral infarction. On an ECG gating scan, his Agatston score was 215 (defined as moderate class). On a nongating scan, coronary calcium deposits (white arrows) were detected in seven slices (Ca-slice\# $=7$, moderate class), and Weston scores were summed up to 7 (left anterior descending artery, 3; left circumflex artery, 2; and right coronary artery, 2). 
A

\begin{tabular}{|c|c|c|c|c|c|c|c|c|c|c|c|c|c|c|}
\hline & & & & & & & & & & & \multicolumn{4}{|c|}{ Карра $=0.134(0.079-0.189)$} \\
\hline & \multicolumn{13}{|c|}{ Weston score } & \multirow{2}{*}{ Total } \\
\hline Agatston & 0 & 1 & 2 & 3 & 4 & 5 & 6 & 7 & 8 & 9 & 10 & 11 & 12 & \\
\hline None: 0 & 29 & 2 & 1 & 0 & 0 & 0 & 0 & 0 & 0 & 0 & 0 & 0 & 0 & 32 \\
\hline Mild: 1-99 & 6 & 4 & 14 & 6 & 14 & 1 & 2 & 1 & 1 & 0 & 0 & 0 & 0 & 49 \\
\hline Moderate: $100-400$ & 0 & 0 & 2 & 2 & 13 & 4 & 8 & 8 & 7 & 1 & 0 & 0 & 0 & 45 \\
\hline Severe: $>400$ & 0 & 0 & 0 & 0 & 2 & 1 & 3 & 3 & 5 & 12 & 6 & 4 & 1 & 37 \\
\hline Total & 35 & 6 & 17 & 8 & 29 & 6 & 13 & 12 & 13 & 13 & 6 & 4 & 1 & 163 \\
\hline Weston class & None & & $\begin{array}{c}M \\
1 .\end{array}$ & & & & & & & & & & & \\
\hline
\end{tabular}

B

\begin{tabular}{|l||c|c|c|c||c|}
\hline \multicolumn{1}{|c||}{ Weston } & $\begin{array}{c}\text { None: } \\
0\end{array}$ & $\begin{array}{c}\text { Mild: } \\
1-3\end{array}$ & $\begin{array}{c}\text { Moderate: } \\
4-8\end{array}$ & $\begin{array}{c}\text { Severe: } \\
9-12\end{array}$ & Total \\
\hline Agatston & 29 & 3 & 0 & 0 & 32 \\
\hline None: 0 & 6 & 24 & 19 & 0 & 49 \\
\hline Mild: $1-99$ & 0 & 4 & 40 & 1 & 45 \\
\hline Moderate: $100-400$ & 0 & 0 & 15 & 22 & 37 \\
\hline Severe: $>400$ & 35 & 31 & 74 & 23 & 163 \\
\hline Total & & 0 & & & \\
\hline
\end{tabular}

Kappa $=0.604(0.511-0.696), P<0.0001$
C

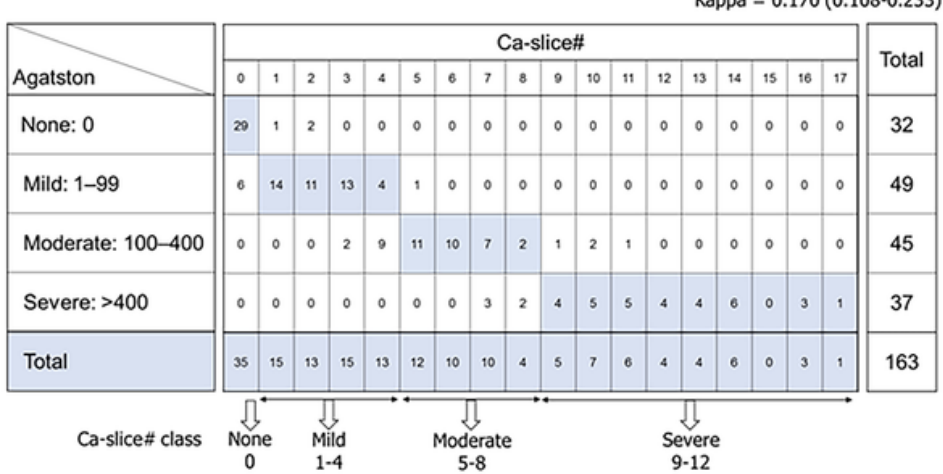

D

\begin{tabular}{|l||c|c|c|c||c|}
\hline \multicolumn{1}{|c||}{ Ca-Slice\# } & $\begin{array}{c}\text { None: } \\
0\end{array}$ & $\begin{array}{c}\text { Mild: } \\
1-3\end{array}$ & $\begin{array}{c}\text { Moderate: } \\
4-8\end{array}$ & $\begin{array}{c}\text { Severe: } \\
9 \leq\end{array}$ & Total \\
\hline Agatston & 29 & 3 & 0 & 0 & 32 \\
\hline None: 0 & 6 & 38 & 5 & 0 & 49 \\
\hline Mild: $1-99$ & 0 & 2 & 39 & 4 & 45 \\
\hline Moderate: $100-400$ & 0 & 2 & 5 & 32 & 37 \\
\hline Severe: $>400$ & 35 & 43 & 49 & 36 & 163 \\
\hline Total & & $0.794(0.720-0.869)$ & $P<0.0001$ & \\
\hline
\end{tabular}

Kappa $=0.794(0.720-0.869), P<0.0001$

\section{Figure 2}

Agreements of classes between each visual score and Agatston score Distribution of Weston and Agatston score classes (A). Weston scores were classified according to the Agatston score class with the greatest frequency; good agreement of classes was thereby obtained between the Weston and Agatston scores (B). Distribution of the total number of slices containing calcium (Ca-slice\#) and Agatston score classes (C). Similarly, Ca-slice\# scores were classified by grouping the Agaston score class with the greatest frequency as none (0), mild (1-4), moderate (5-8), and severe (9-12). Good agreement of classes was obtained between the Ca-slice\# and Agatston score (D). 
A

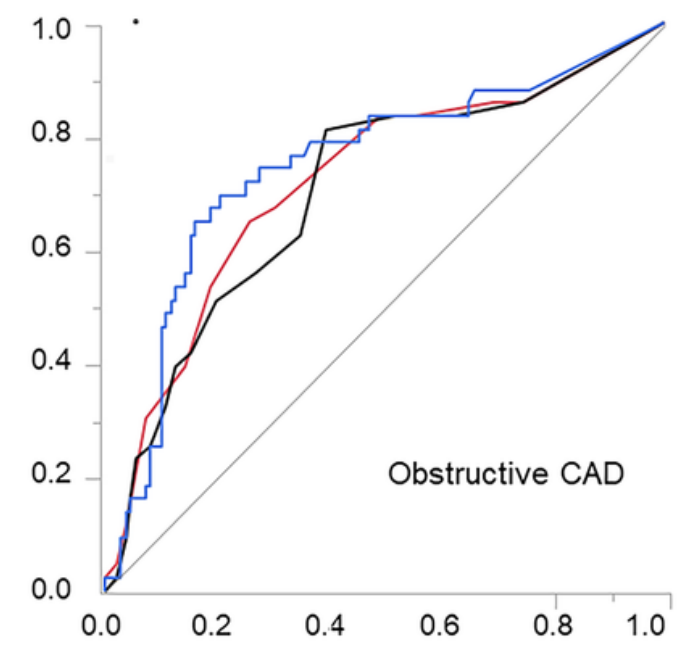

\begin{tabular}{|c|c|c|}
\hline \multicolumn{3}{|c|}{ c-statistics ( $95 \% \mathrm{CI})$} \\
\hline Agatston score & 0.750 & $(0.646-0.831)$ \\
\hline Weston score & 0.721 & $(0.618-0.805)$ \\
\hline Ca-slice\# & 0.706 & $(0.603-0.798) *$ \\
\hline
\end{tabular}

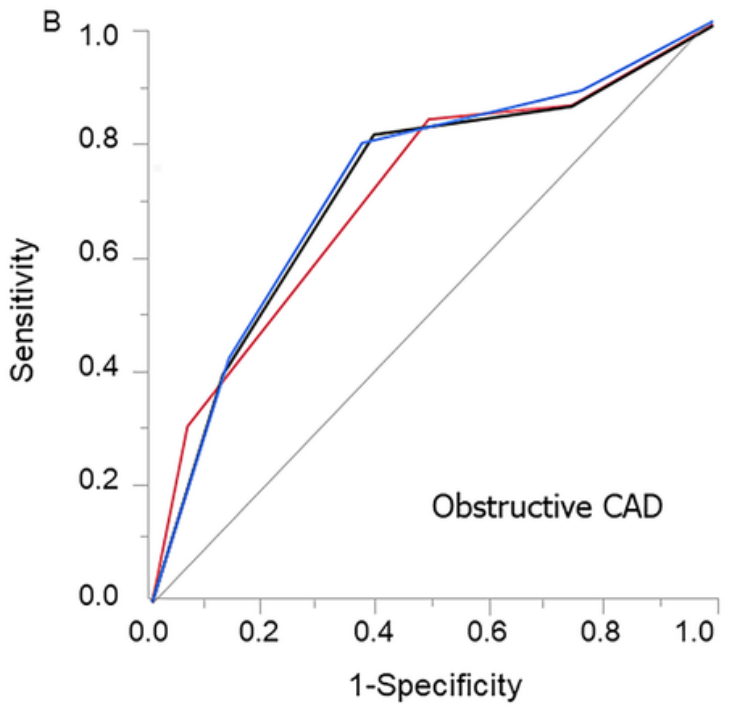

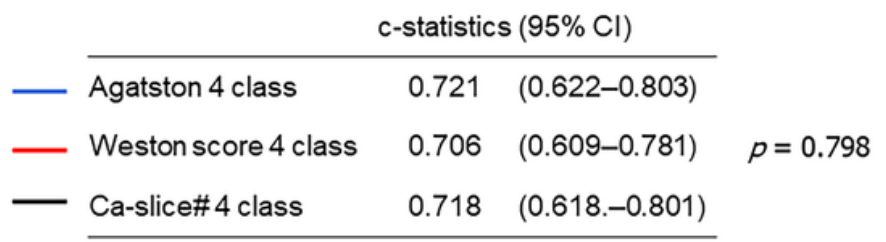

\section{Figure 3}

Receiver-operator characteristics analyses to detect obstructive CAD Obstructed CAD was found in 43 $(27 \%)$ of 155 patients. Comparison of c-statistics of Agatston score, Weston score, and Ca-slice\# (A). Comparison of c-statistics of classes of Agatston score, Weston score, and Ca-slice\# (B). ${ }^{*} \mathrm{C}$-statistics were significantly lower than those from the Agatston score (difference, $0.044 ; 95 \% \mathrm{Cl} 0.008-0.080 ; \mathrm{P}=$ 0.016).

\section{Supplementary Files}

This is a list of supplementary files associated with this preprint. Click to download.

- easyCACMulticenterlJCVIsupple.pdf 